\title{
Novel Modeling of Somatosensory Evoked Potentials for the Assessment of Spinal Cord Injury
}

\author{
H. Mir, Senior Member, IEEE, H. Al-Nashash, Senior Member, IEEE, J. Kortelainen, Member, IEEE, and A. All
}

\begin{abstract}
Objective: Previous work has shown that differences in the somatosensory evoked potential (SEP) signals between a normal spinal pathway and spinal pathway affected by spinal cord injury (SCI) provide a means to study the degree of injury. This paper proposes a novel quantitative SCI assessment method using time-domain SEP signals. Methods: A pruned and unstructured fit between SEP signals from a normal spinal pathway and a spinal pathway affected by SCI is developed using methods inspired by recent results in sparse reconstruction theory. The coefficients from the resulting fit are used to develop a quantitative assessment of SCI that is tested on actual SEP signals collected from rodents that have been subjected to partial and complete spinal cord transection. Results: The proposed method provides a rich parametric measure that integrates SEP amplitude, time latency, and morphology, while exhibiting a high degree of correlation with existing subjective and quantitative SCI assessment methods. Conclusion: The proposed SCI encapsulates a model of the injury to quantify SCI. Significance: The proposed SCI quantification method may be used to complement existing SCI assessment methods.
\end{abstract}

Keywords -Somatosensory Evoked Potential (SEP), Spinal Cord Injury (SCI), Sparse Reconstruction

\section{INTRODUCTION}

All bioelectrical signals between the limbs and the brain must pass through the spinal cord. Consequently, any spinal cord injury (SCI) will degrade signal transmission, resulting in loss or impairment of sensory and/or motor function [1]. Globally, millions of individuals are affected by SCI, with tens of thousands of new cases arising each year [2]. The prevalence of SCI motivates the need to develop novel diagnostic approaches to facilitate the development of novel therapeutic methods. In addition to early detection or diagnosis, monitoring of SCI in the clinical recovery and injury management phases is of paramount importance. This is required to assess the effectiveness of potential therapeutic procedures [3]. In addition to the standard rehabilitation regimes used to help SCI patients, there are several medical and surgical procedures that may be used to mitigate the effects of injury. Indeed, even a small number of spared spinal nerve fibers coupled with immediate diagnosis can greatly improve the quality of life of patients with SCI. There has also been recent work [4] on the functional plasticity and neuroanatomical reorganization and changes of pathways that take place immediately after injury.

H. Mir and H. Al-Nashash are with the Department of Electrical Engineering, American University of Sharjah, UAE.

H. Al-Nashash is also with A. All at the SINAPSE Institute, National University of Singapore, Singapore.

J. Kortelainen is with the Physiological Signal Analysis Team, Center for Machine Vision and Signal Analysis, and the Department of Clinical Neurophysiology, University of Oulu, Finland.
Clearly, there is a pressing need for an accurate, repeatable, and noninvasive assessment method to quantify the degree of SCI.

Modalities used for estimating the level of SCI are dominated by electrophysiological [5],[6] and imaging [7],[8] techniques. While imaging approaches provide information about the location and the anatomical damage of the injury, they do not provide quantitative information about the functional integrity of the spinal cord itself.

By contrast, electrophysiological techniques that measure the evoked potential can provide information about the functional integrity of the spinal cord. The evoked potential is the electrophysiological response of the neural system to an external stimulus. The somatosensory evoked potential (SEP) is the cortical signal recorded from the brain in response to electrical stimulation usually given to the median nerve at the wrist or the posterior tibial nerve at the ankle [9]. The SEP is of particular interest to researchers when evaluating the ongoing neurophysiological changes throughout the post-SCI period. It was shown in [10] that the similarity between a SEP signal from a healthy spinal pathway (which in the event of thoracic SCI can be obtained by forelimb stimulation) and a SEP signal from an injured spinal pathway (which can be obtained by hindlimb stimulation) can be used as an objective measure of the severity of SCI, thus providing a complementary measure to qualitative behavioral based assessments in rodents such as the clinical examination of Basso, Beattie, and Bresnahan (BBB) [11].

Existing SCI assessment measures tend to be based on the SEP peak amplitude and/or time latency, without regard to the overall signal morphology. In this regard, the measure in [10] accounts for amplitude/power and morphological similarity between a baseline SEP signal from a healthy spinal pathway and a SEP signal from a SCI affected spinal pathway, though time latency is not explicitly considered. This paper builds upon the work in [10]. In particular, this paper proposes to model the morphology of the SEP signal from the SCI affected spinal pathway as a transformation of the baseline SEP signal that consists of a superposition of time-shifts and amplitude scales of the baseline SEP signal. It is shown that the proposed method results in a set of time-shifts and amplitude scales which encapsulate information that is directly related to the severity of the injury. A measure is also developed that integrates both SEP amplitude and time latency into a single quantity.

The rest of this paper is organized as follows: Section II describes the protocol for collecting the SEP signals from rodents. Section III describes the proposed SCI assessment method. Section IV presents the results of applying the method 
in Section III to the data described in Section II.

\section{Protocol and Data Collection}

Animals: Female adult Sprague-Dawley rats weighing between 200-225g were used. Two types of thoracic spinal transection, namely T7/T8 left hemitransection and T8 complete transection, were performed. All procedures were approved by the Institutional Animal Care and Use Committee (IACUC) at the National University of Singapore.

Electrode Implantation: To induce general anesthesia, the rodents were anesthetized intraperitoneally with a mixture of ketamine $(75 \mathrm{mg} / \mathrm{kg})$ and xylazine $(10 \mathrm{mg} / \mathrm{kg})$ at $0.2 \mathrm{ml} / 100 \mathrm{~g}$. A midline incision through the skin of the head was made and the skull was exposed and cleaned. The rodents were implanted with transcranial stainless steel screw electrodes corresponding to forelimb and hindlimb somatosensory cortices, such that they made light contact with the dura mater without compressing the brain. A micro drill was used to create burr holes with the bregma and lambda used as references to fix the positions of the electrodes. Carboxylate dental cement was subsequently applied to hold the screw electrodes in place and seal the exposed skull.

Injury: General anaesthesia as reported above was used during all surgical procedures. Limb withdrawal to pinch stimuli and the corneal reflex were monitored in order to ascertain that the animal was adequately anesthetised. Laminectomy was performed to expose the dorsal surface of the spinal cord. Care was taken to maintain the integrity of the dura mater. A stainless steel disposable scalpel was used to carry out the transection injuries.

Post-operative Care: Analgesic Buprenorphine $(0.06 \mathrm{mg} / \mathrm{kg})$ and antibiotic Gentamicin $(8 \mathrm{mg} / \mathrm{kg})$ at $0.2 \mathrm{ml} / 100 \mathrm{~g}$ volume were administered sub-cutaneously twice a day for five days following transection injury. The rodents' bladders were expressed twice daily until they regained the ability to urinate.

Electrophysiological recording: A Tucker-Davis Technologies (TDT) workstation was used to record the SEP signals. The SEP baseline recordings were carried out one week after electrode implantation, and post-injury SEP recordings were performed one week after inflicting SCI. The median and tibial nerves were stimulated using stainless steel subdermal needle electrodes, and SEP signals were recorded from the implanted electrodes at a sampling frequency of $4882 \mathrm{~Hz}$. The stimulation of each limb was carried out using 156 consecutive pulses with an amplitude of $3.5 \mathrm{~mA}$ and a duration of $200 \mu \mathrm{sec}$. During SEP recording, a mixture of $1.5 \%$ isoflurane and $98 \%$ oxygen at a flow rate of $1.3 \mathrm{~L} / \mathrm{min}$ was supplied through an isoflurane vaporizer. The anesthesia was maintained through a rodent-sized anesthesia mask, connected to a diaphragm with a C-pram circuit designed to administer and evacuate the gas. Rodents were moved onto a heating pad to maintain a body temperature of $37 \pm 0.5^{\circ} \mathrm{C}$.

BBB analysis: A 21 score BBB locomotion rating scale was used to assess the motor behavior of the rodents before and after injury. Rats were placed in a $90-\mathrm{cm}$ plastic open field for 4 minutes. Two blinded examiners proficient in the method evaluated and scored the animals individually.

\section{SCI Assessment Method}

Let $b_{n}, n=0 \ldots N-1$ denote the baseline SEP signal collected from a healthy spinal pathway, and let $d_{n}, n=$ $0 \ldots N-1$ denote the SEP signal collected from a spinal pathway that has been subjected to SCI. It is assumed that $d_{n}$ can be modeled as the output of a moving average system excited by $b_{n}$. That is, $d_{n}$ can be modeled as a superposition of time-shifted and amplitude scaled versions of $b_{n}$. From a systems perspective, $b_{n}$ can be viewed as the input to a finite-impulse-response filter with coefficients $h_{n}$, and $d_{n}$ is the resulting filter output that is obtained by convolving $b_{n}$ with $h_{n}$, that is:

$$
\begin{aligned}
d_{n} & \approx h_{-M} b_{n+M}+h_{-M+1} b_{n+M-1}+\cdots+h_{M} b_{n-M} \\
& =\sum_{m=-M}^{M} h_{m} b_{n-m}
\end{aligned}
$$

where $M$ is the maximum time-shift. Now define the vectors

$$
\mathbf{h}=\left[\begin{array}{c}
h_{-M} \\
h_{-M+1} \\
\vdots \\
h_{M}
\end{array}\right], \mathbf{b}_{n}=\left[\begin{array}{c}
b_{n+M} \\
b_{n+M-S} \\
\vdots \\
b_{n-M}
\end{array}\right]
$$

Then (1) can be compactly expressed as:

$$
d_{n} \approx \mathbf{h}^{T} \mathbf{b}_{n}
$$

The solution for the unknown model parameter vector $\mathbf{h}$ is obtained by solving the least-squares optimization problem:

$$
\underset{\mathbf{h}}{\arg \min } \sum_{n=0}^{N-1}\left\|d_{n}-\mathbf{h}^{T} \mathbf{b}_{n}\right\|_{2}^{2}
$$

which yields the solution:

$$
\mathbf{h}=\left[\sum_{n=0}^{N-1} \mathbf{b}_{n} \mathbf{b}_{n}^{T}\right]^{-1}\left[\sum_{n=0}^{N-1} d_{n} \mathbf{b}_{n}\right]
$$

The $m$ th element of $\mathbf{h}$ has the effect of shifting $b_{n}$ by $m-M-$ 1 time units and scaling the amplitude by $h_{m}$. It is apparent that if $d_{n}$ is similar to $b_{n}$ (implying a low level of SCI), then $\mathbf{h}$ should consist of mostly zero valued elements except for the $(M+1)$ th element (corresponding to zero time-shift) which should have a value around one (i.e. $\mathbf{h}$ is essentially a unit impulse). On the other hand, if $d_{n}$ is very dissimilar to $b_{n}$ (implying a high level of SCI), then the structure of $\mathbf{h}$ will be more complex, since combining a variety of time-shifts and amplitude scales of $b_{n}$ is needed to accurately model $d_{n}$.

Figures 1(a)-(d) show how various forms of the elements $h_{m}$ of the vector $\mathbf{h}$ affect the transformation of $b_{n}$ to $d_{n}$. In Figure 1(a), the only non-zero element in $\mathbf{h}$ has a value of 1.3 and is located at a time-shift of 0. Hence, $d_{n}$ has no timeshift relative to $b_{n}$, but the amplitude of $d_{n}$ has increased by a factor of 1.3. In Figure 1(b), the only non-zero element in $h$ has a value of 0.9 and is located at a time-shift of -10 . Hence, $d_{n}$ time advanced (shifted to the left) relative to $b_{n}$ by 10 time samples, and the amplitude of $d_{n}$ is decreased by a factor of 0.9. Similarly, in Figure 1(c), the only non-zero element in $\mathbf{h}$ has a value of 2 and is located at a time-shift of +10 . Hence, 

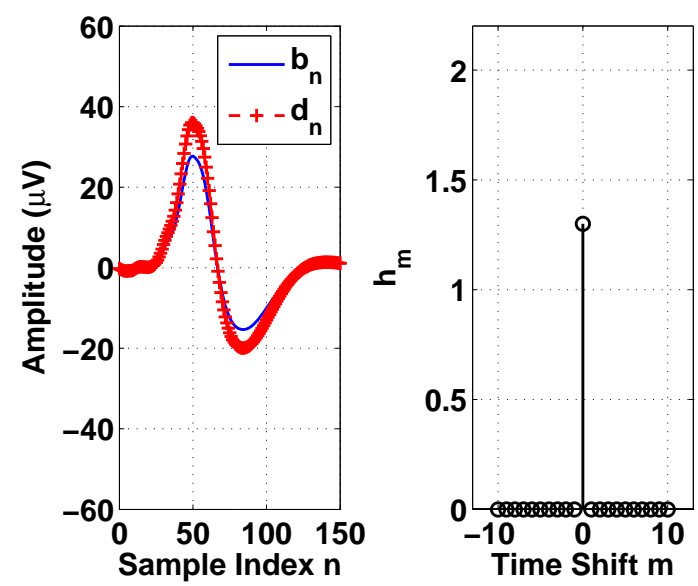

(a)

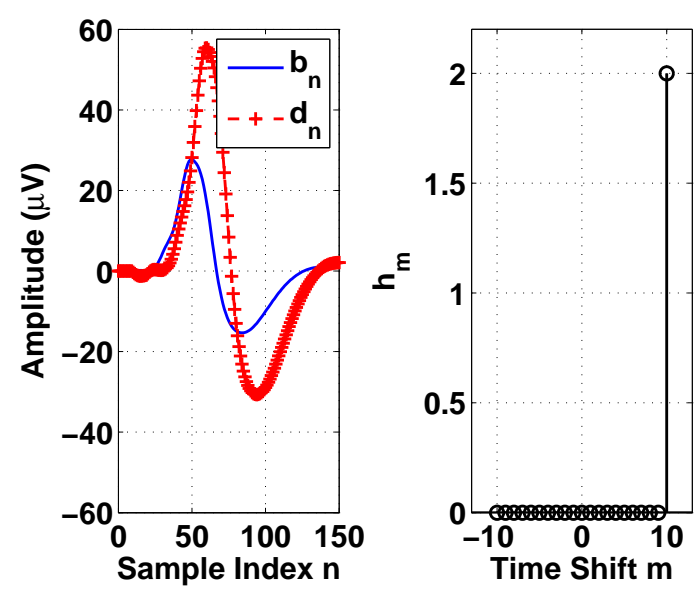

(c)

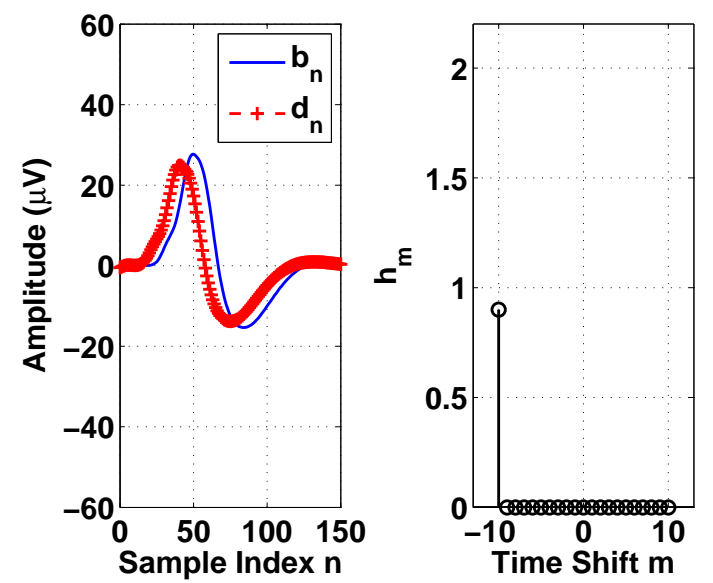

(b)

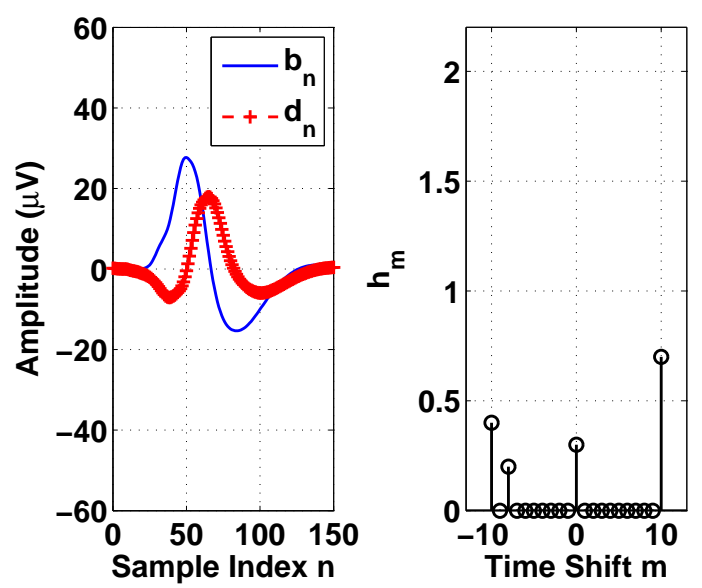

(d)

Fig. 1: (a)-(d) The effect of various forms of $h_{m}$ on transforming $b_{n}$ into $d_{n}$.

$d_{n}$ time delayed (shifted to the right) relative to $b_{n}$ by 10 time samples, and the amplitude of $d_{n}$ is increased by a factor of 2. Finally, Figure 1(d) depicts a situation in which there are several non-zero elements of $\mathbf{h}$ located at various time-shifts. The shape of $d_{n}$, therefore, is not simply a time-shift and/or amplitude scale of $b_{n}$, resulting in the emergence of a more complex structure.

The solution for $\mathbf{h}$ in (3) is the conventional least squares fit between $b_{n}$ and $d_{n}$. However, (3) suffers from the problems of overfitting and sensitivity to effects that cause $d_{n}$ to deviate from the model in (1). That is, the parameter vector $\mathbf{h}$ contains excess degrees of freedom; indeed, though only a subset of time-shifts from $-M$ to $+M$ may be needed to model the transformation, (3) will attempt to use all possible time-shifts. As an illustration, consider again the signals $b_{n}$ and $d_{n}$ in Figure 1(d). Inserting the values for $b_{n}$ and $d_{n}$ in (3) will indeed yield exactly the same set of values for the elements of $\mathbf{h}$ shown in the right-hand panel of Figure 1(d). Now consider the signals $b_{n}$ and $d_{n}$ in Figure 2(a). While the signal $b_{n}$ is the same as in Figure 1(d), the signal $d_{n}$ has been perturbed by a small amount of random noise. While this random perturbation does not have much visual effect on $d_{n}$ compared to Figure 1(d), the resulting set of coefficients generated using (3) is markedly different, as can be seen in Figure 2(b).

One possibility to alleviate this problem is to use some form of structured pruning of $\mathbf{h}$. That is, the model in (1) could be modified in order to include only a subset of the time-shifts from $-M$ to $+M$, thereby limiting the degrees of freedom. However, there is no apparent way to methodically choose the appropriate subset of time-shifts. A better approach would thus be to use unstructured pruning in which the fitting method automatically selects the most appropriate subset of time-shifts. This objective can be mathematically expressed using the $l_{0}$-norm of $\mathbf{h}$, denoted as $\|\mathbf{h}\|_{0}$, which is simply the number of non-zero elements in $\mathbf{h}$. The $l_{0}$-norm can be used to promote a sparse solution for $\mathbf{h}$, since attempting to minimize $\|\mathbf{h}\|_{0}$ effectively minimizes the degrees of freedom (and hence the size of the subset of time-shifts) that are used. This minimization, however, must be subject to a goodnessof-fit constraint so that the error between the actual and fitted data resulting from a sparse $\mathbf{h}$ does not exceed some tolerance level $\delta$. The formal mathematical expression for the desired 


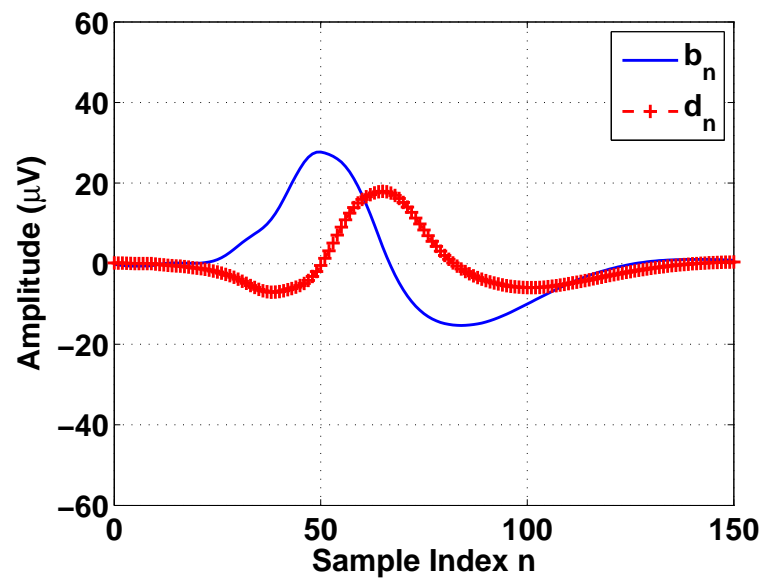

(a)

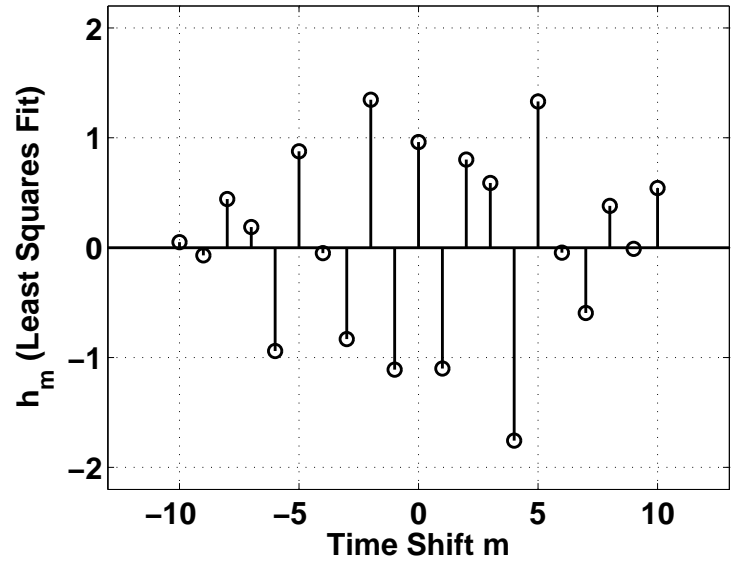

(b)

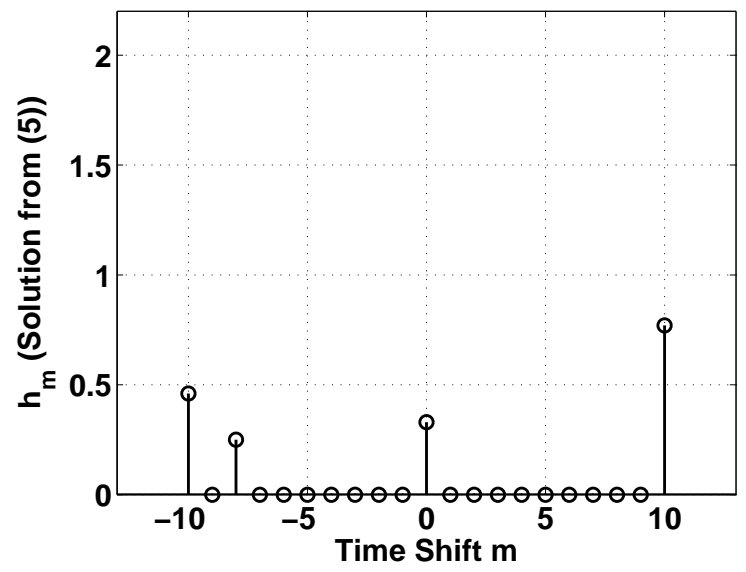

(c)

Fig. 2: (a) $b_{n}$ is the same as in Figure 1(d) but $d_{n}$ has been perturbed by random noise. (b) Example of over-fitting using conventional least squares (3). (c) Example of fitting improvement using (5).

optimization problem thus becomes:

$$
\underset{\mathbf{h}}{\arg \min }\|\mathbf{h}\|_{0} \ni \sum_{n=0}^{N-1}\left\|d_{n}-\mathbf{h}^{T} \mathbf{b}_{n}\right\|_{2}<\delta
$$

The optimization problem in (4) is quite difficult to solve due to the presence of the $l_{0}$-norm in the objective function. To alleviate this problem, consider substituting the $l_{0}$-norm with the $l_{1}$-norm, so that the objective function instead becomes $\|\mathbf{h}\|_{1}$. As such, (5) may be recast as:

$$
\underset{\mathbf{h}}{\arg \min }\|\mathbf{h}\|_{1} \ni\|\mathbf{B h}-\mathbf{d}\|_{2}<\delta
$$

where

$$
\mathbf{B}=\left[\begin{array}{c}
\mathbf{b}_{0}^{T} \\
\mathbf{b}_{1}^{T} \\
\vdots \\
\mathbf{b}_{N-1}^{T}
\end{array}\right], \mathbf{d}=\left[\begin{array}{c}
d_{0} \\
d_{1} \\
\vdots \\
d_{N-1}
\end{array}\right]
$$

This substitution in $(5)$ of the $l_{0}$-norm with the $l_{1}$-norm is motivated by recent developments in the field of compressed sensing (CS) [12], which is closely related to sparse re- construction. CS is a novel signal processing technique for solving an underdetermined system whose underlying solution is known to be sparse. It may be noted that in (5), there are typically more measurements than unknowns, leading to an overdetermined rather than an undetermined system. Hence, the usual requirement from CS theory that the columns of the matrix $\mathbf{B}$ should be uncorrelated can be relaxed. As such, while (5) does not have a closed form solution like its counterpart (2), various efficient numerical methods such as Chaining Pursuit [13], Orthogonal Matching Pursuit [14], and Compressive Sampling Matching Pursuit (CoSaMP) [15],[16] that have been developed for CS problems can be applied. Due to its relatively low computational complexity, CoSaMP is used in this paper to approximate the solution to (5). The operation of CoSaMP is show in the Algorithm inset and can be summarized as follows:

- Line 1: Use a trivial initialization for $\mathbf{h}$, resulting in the initial error $\mathbf{e}$ being equal to the desired signal $\mathbf{d}$; initialize the loop counter $k$

- Line 2: Continue loop until error magnitude decreases to a desired level $\delta$ 
- Line 3: For a desired sparsity level $s$, store the indices of the $s$ largest elements of the proxy signal $\mathbf{B}^{T} \mathbf{e}$ in the set $\mathcal{I}_{1}$.

- Line 4: Store the indices of the non-zero elements in the current estimate of $\mathbf{h}$ in the set $\mathcal{I}_{2}$.

- Line 5: Merge the newly identified indices $\mathcal{I}_{1}$ with the existing indices $\mathcal{I}_{2}$ into the set $\mathcal{I}$.

- Line 6: Update the estimate of $\mathbf{h}$ to retain the $s$ largest elements at the appropriate indices using the least squares estimate $\mathbf{B}(:, \mathcal{I})^{\#} \mathbf{d}$

- Line 7: Update the estimate of the error e

- Lines 8-9: Increment the counter / end of loop.

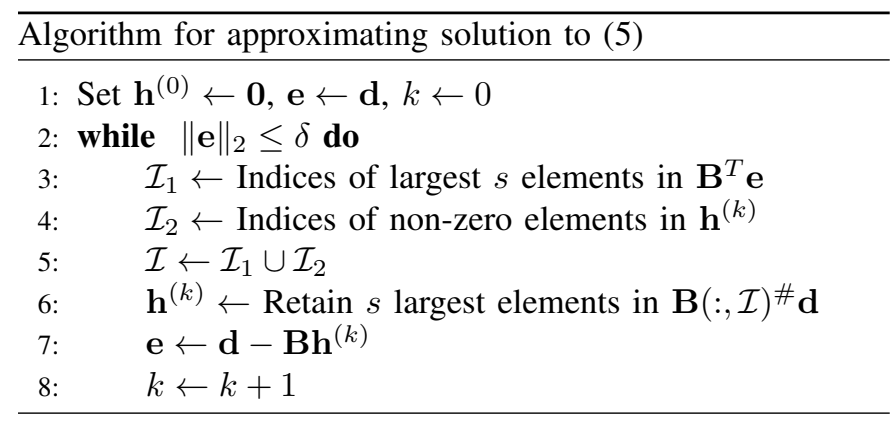

Figure 2(c) shows the elements of $\mathbf{h}$ resulting from solving (5). It can be seen that the set of coefficients is nearly identical to that in the right hand panel of Figure 1(d), which suggests that (5) is robust to model perturbations. Since (5) generates a solution for $\mathbf{h}$ that automatically selects only the significant subset of time-shifts in an unstructured manner, the resulting solution for $\mathbf{h}$ may be viewed as encapsulating a model for the injury. For mild forms of SCI, $d_{n}$ will likely be well approximated as a shifted and scaled version of $b_{n}$, and $\mathbf{h}$ should thus essentially be a unit impulse. However, a more severe form of SCI will cause $d_{n}$ to be dissimilar to $b_{n}$, necessitating the use of increased degrees of freedom, resulting in a solution for $\mathbf{h}$ that contains more non-zero elements. As such, the sparsity of $\mathbf{h}$ provides an indication of the level of SCI. Moreover, the specific placement and magnitude of the non-zero elements in $\mathbf{h}$ provides a model of the injury. Whereas $b_{n}$ is considered to be from a single reference neural path with unit magnitude, $d_{n}$ is formed from multiple paths (with respect to the reference path of $b_{n}$ ) due to the injury, each of which exhibits a time-shift and scaling effect. The output of these paths, when superposed at the cortex, forms the signal $d_{n}$. As such, the placement of the non-zero elements of $\mathbf{h}$ provides information related to latency, while the magnitude of the non-zero elements of $\mathbf{h}$ provides information related amplitude/power. The vector $\mathbf{h}$ thus provides a richer description of the injury.

In order to compare the proposed method with existing assessment techniques, however, it is necessary to condense $\mathbf{h}$ into a single number. As discussed earlier, the sparsity or similarity of $\mathbf{h}$ to a unit impulse provides an indication of the level of SCI. The proximity of $\mathbf{h}$ to a unit impulse will be quantified as:

$$
I_{D}=\alpha J\left(k^{*}\right)+(1-\alpha)\left|\frac{k^{*}}{M}\right|
$$

where $0 \leq \alpha \leq 1$, and

$$
k^{*}=\underset{k \in[-M, M]}{\arg \min } J(k)
$$

and

$$
J(k)=\frac{\sum_{m=-M}^{M}\left|h_{m}-\delta_{m-k}\right|}{1+\sum_{m=-M}^{M}\left|h_{m}\right|}
$$

In this manner, $I_{D}$, which will be termed as the impulse distance, attempts to measure the similarity of $\mathbf{h}$ to a shifted unit impulse with a penalty for impulse locations away from $m=0$. Note also that in (6), $J\left(k^{*}\right)$ and $\left|\frac{k^{*}}{M}\right|$ are assigned a weighting term of $\alpha$ and $1-\alpha$, respectively. While $J\left(k^{*}\right)$ is associated with the SEP amplitude characteristics, the term $\left|\frac{k^{*}}{M}\right|$ is associated with the time characteristics. Thus, the weighting of each term may be suitably adjusted through the value of $\alpha$ in order to emphasize/de-emphasize the amplitude and/or time characteristics. In Section IV, the value of $\alpha$ is set to 0.5 in order to given equal weighting to the two terms.

\section{RESUlts}

All SEP signals used in this paper are the ensemble average of 156 single-trial signals. Figure 3 shows the baseline hindlimb SEP signal $b_{n}$ and SCI affected hindlimb SEP signal $d_{n}$ for the cohort used in this paper. The top row of Figure 3 shows the SEP signals of rodents that were inflicted with partial SCI (spinal hemitransection), while the bottom row of Figure 3 shows the SEP signals of rodents that were inflicted with the more severe complete SCI (full spinal transection). As discussed in the previous section, the nature of the vector $h$ resulting from solving (5) reflects the level of SCI. Towards this end, $\delta$ was set to $10^{-3}$, and $M$ was set to 20 samples. The choice for $M$ was determined empirically as it was found that further increasing the value for $M$ did not provide any improvement to the quality of the fit.

Figure 4 shows plots of the elements $\left\{h_{-M}, \ldots, h_{M}\right\}$ of $\mathbf{h}$ obtained from solving (5), where the time-shift in samples has been converted to time in milliseconds. The top row of plots shows the elements of $\mathbf{h}$ for rodents with spinal hemitransection, while the bottom row shows the elements of $\mathbf{h}$ for rodents with full spinal transection. The significance of an element is primarily based on its amplitude relative to the other elements, since the amplitude is proportional to the contribution that the corresponding amplitude scaled/time shifted version of $b_{n}$ makes to the overall shape of the $d_{n}$. Moreover, if $b_{n}$ and $d_{n}$ are similar (as is the case for moderate $\mathbf{S C I}$ ), it is expected that $\mathbf{h}$ will be similar to an impulse function and contain only one significant element. Conversely, if $b_{n}$ and $d_{n}$ are dissimilar (as is the case for severe SCI), it is expected that $\mathbf{h}$ will contain several elements that are all relatively small in value. It can be seen that for the top row in Figure 4, the elements of $\mathbf{h}$ contain only one significant element at a small or zero time-shift, which implies that $d_{n}$ is largely similar in shape and temporal alignment to $b_{n}$, as can be verified by visually examining the corresponding SEP signals in the top row of Figure 3. This is consistent with 

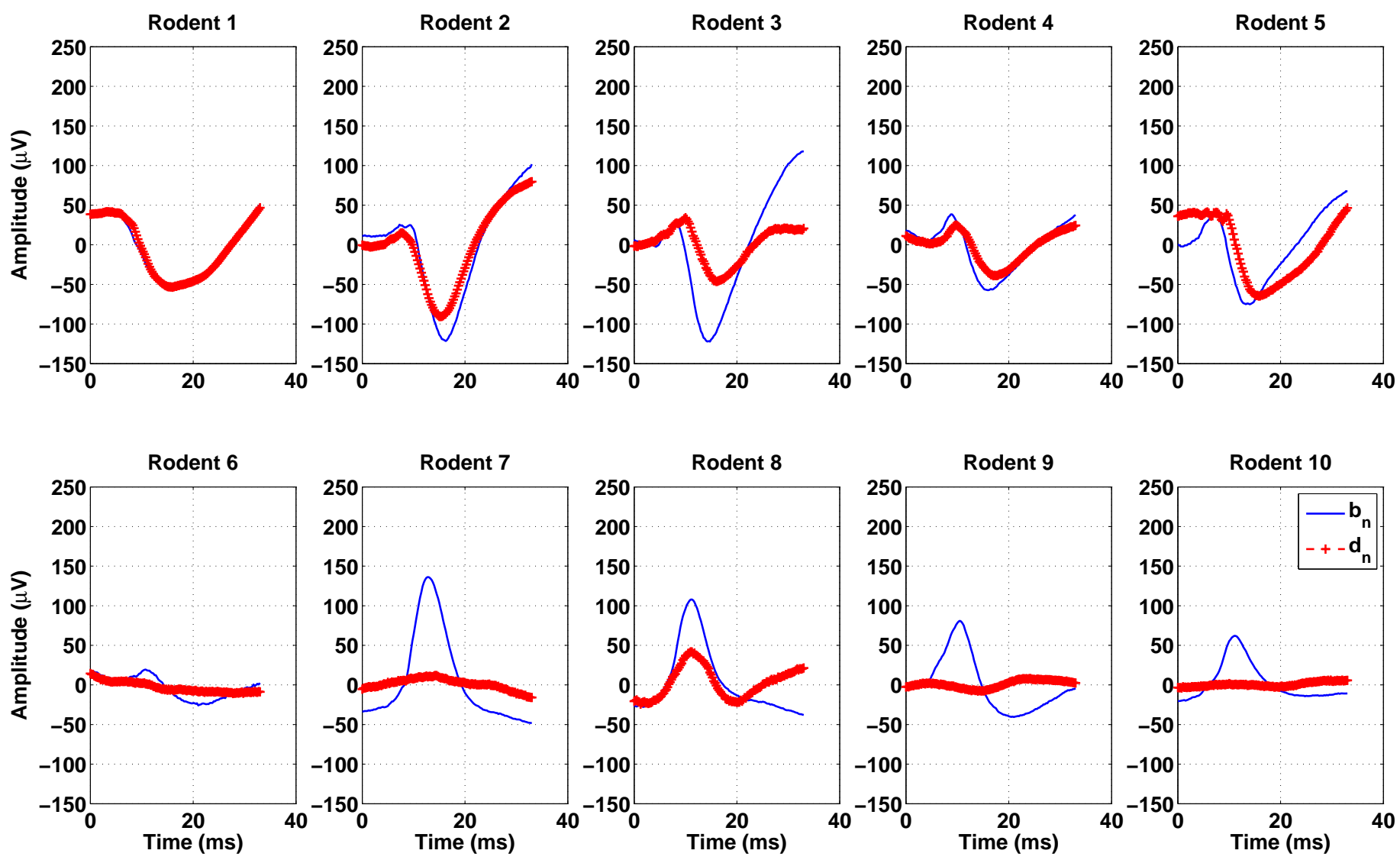

Fig. 3: Baseline hindlimb SEP and SCI affected hindlimb SEP signal for rodents with spinal hemitransection (top row) and full spinal transection (bottom row).

the fact that the rodents in this case have been subjected to a spinal hemitransection, rather than a full spinal transection. On the other hand, it can be seen that for the bottom row in Figure 4, several elements of $\mathbf{h}$ with small values are located at a variety of time-shifts, implying $d_{n}$ to be largely dissimilar in shape and temporal alignment to $b_{n}$. This can again be verified by examining the corresponding SEP signals in the bottom row of Figure 3. It is also consistent with the fact that the rodents in this case have been subjected to a full spinal transection. Thus, when $\mathbf{h}$ consists of only a few significant non-zero elements located at time-shifts close to zero, the degree of SCI is relatively mild. However, when $\mathbf{h}$ consists of many relatively small non-zero elements located at various time-shifts, the degree of SCI is more severe.

To verify the proposed methodology for quantifying SCI, the value of the impulse distance $I_{D}$ in (6) will be compared with both the quantitative assessment technique of spectral coherence [10] as well as the qualitative assessment method of BBB. Figure 5(a) shows the impulse distance for the rodent cohort while Figure 5(b) shows the spectral coherence for the rodent cohort. The magnitude-squared spectral coherence is defined as:

$$
C(f)=\frac{\left|S_{b d}(f)\right|^{2}}{S_{b b}(f) S_{d d}(f)}
$$

where $S_{b d}(f)$ is the cross spectral power spectrum of $b_{n}$ and $d_{n}, S_{b b}(f)$ is the power spectrum of $b_{n}$, and $S_{d d}(f)$ is the power spectrum of $d_{n}$. The spectral coherence is computed by integrating $C(f)$ over the half-power bandwidth, which occurs from around 75-225 Hz. It should be noted that a higher impulse distance value corresponds to an $\mathbf{h}$ that is more dissimilar to a unit impulse and thus represents a higher level of injury, and vice-versa. However, a higher spectral coherence value corresponds to a lower level of injury, and vice-versa. As such, it can be seen that both measures depicted in Figures 5(a) and 5(b) in fact agree very well with each other, with an overall Pearson Correlation Coefficient magnitude of 0.93 between the two (obviously an increased number of animals would improve the statistical significance). The major advantage of the proposed method over the spectral coherence, however, is that it provides a closer inspection of the change in morphology of the SEP signal caused by injury. Moreover, quantitative changes in SEP morphology may have useful clinical applications.

Although the subjective BBB test does not provide a direct measure of the accuracy of the proposed method, it will be used in this paper to compare the overall correlation between the opinion of experienced electrophysiologists and the proposed quantitative method. Figure 5(c) shows the BBB scores for the rodent cohort, while Table I shows the Pearson Correlation Coefficient magnitude of the BBB score with the impulse distance and spectral coherence. The general trend of the BBB score is consistent with the impulse distance and spectral coherence values, resulting in the Pearson Correlation 

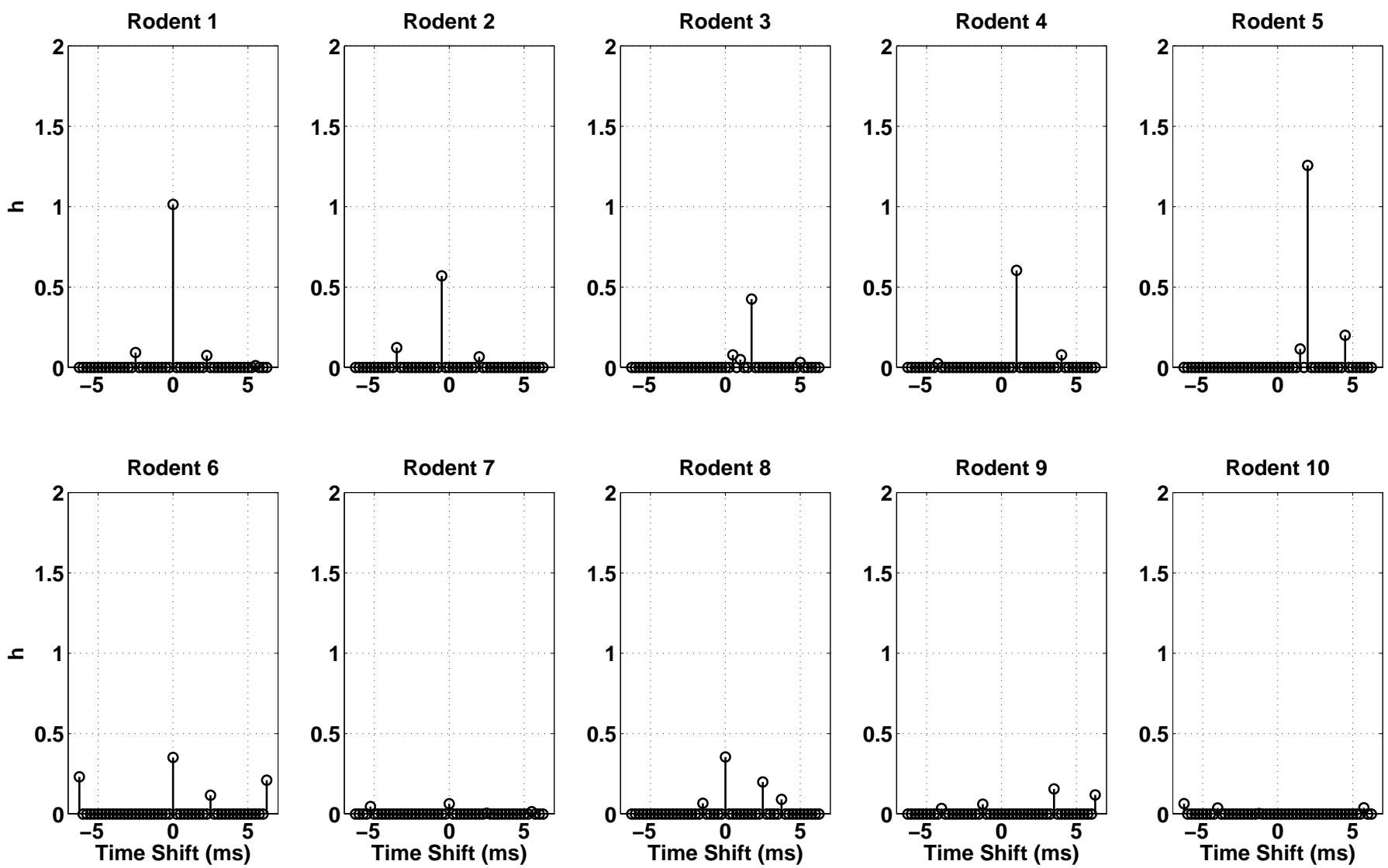

Fig. 4: Elements of $\mathbf{h}$ from solving (5) for rodents with spinal hemitransection (top row) and full spinal transection (bottom row) using the SEP signals in Figure 3.

Coefficient magnitude with both the impulse distance and spectral coherence to be around 0.7 , thus raising confidence about the proposed methodology.

The post-SCI SEP signal in Figure 3 of Rodent 8 appears to show some resilience to the inflicted injury, a fact which is reflected in Figures 5(a)-5(c) across the impulse distance, spectral coherence, and BBB score. On the other hand, the post-SCI SEP signal in Figure 3 of Rodent 3 shows a very modest recovery. The degree of this recovery is more closely reflected in the impulse distance value compared to the score of the BBB complement, for which there is essentially no motor function recovery. As noted in [10], a recovery in the shape of the SEP waveform reflects the health of the spinal pathway.

TABLE I: Pearson Correlation Coefficient magnitude for SCI assessment methods using the SEP signals in Figure 3

\begin{tabular}{|c|c|c|}
\hline & Spectral Coherence & BBB Score \\
\hline \hline Impulse Distance & 0.92 & 0.67 \\
\hline Spectral Coherence & 1 & 0.71 \\
\hline
\end{tabular}

The preceding discussion is based on the availability of a baseline SEP signal which is recorded prior to SCI. This scenario is applicable in a laboratory setting, and is useful in monitoring the effects and progression of SCI. In a clinical setting, however, it is very unlikely that a baseline SEP signal will be available for an SCI patient. It may be noted, however, that SCI most commonly occurs at the cervical and thoracic vertebrae [17]. The spinal pathway from the forelimb to the cortex as such will exhibit injury for cervical SCI, whereas it will remain intact for SCI at or below the thoracic region. Thus, the SCI assessment method proposed in this paper may be applied in a clinical setting to thoracic SCI, wherein the SEP signal recorded in response to forelimb stimulation can serve as a surrogate for the hindlimb baseline SEP signal. This approach was also adopted in [10].

Figure 6 shows the same SCI affected hindlimb SEP signal $d_{n}$ as in Figure 3 along with the baseline forelimb SEP signal $b_{n}$ that was acquired by stimulating the forelimb after inflicting SCI. Figures 7-8 show the elements of $\mathbf{h}$, the impulse distance, and the spectral coherence resulting from using the SEP signals in Figure 6. It can be seen that the results in Figures 7-8 are quite similar to those in Figures 4-5, which lends credence to using the forelimb SEP signal as a surrogate for the baseline hindlimb SEP signal in cases of thoracic SCI. Table II shows the resulting Pearson Correlation Coefficient Magnitudes, which are also similar to those in shown Table I.

A quantifiable measure of the SEP signal morphology that can be related to the level of SCI is useful because it can be easily interpreted, whereas inspecting the signal morphology requires a trained examiner. It may be noted that demyelination is a consequence of nerve injury, which causes reduced propa- 


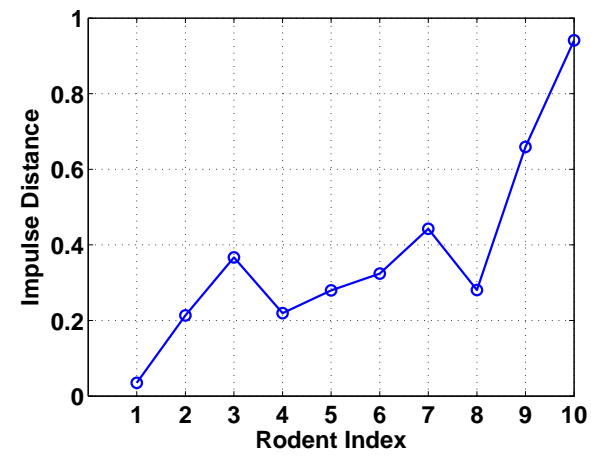

(a)

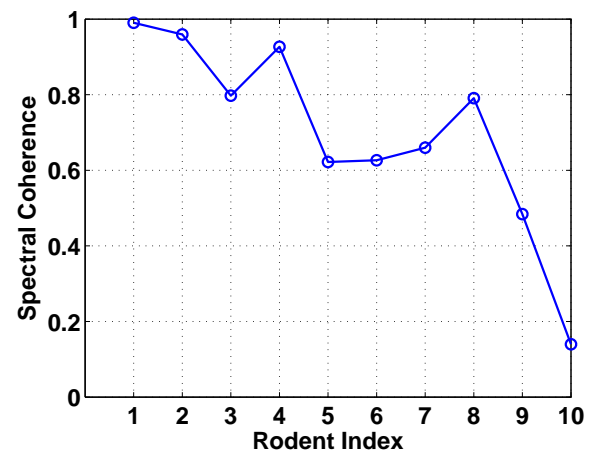

(b)

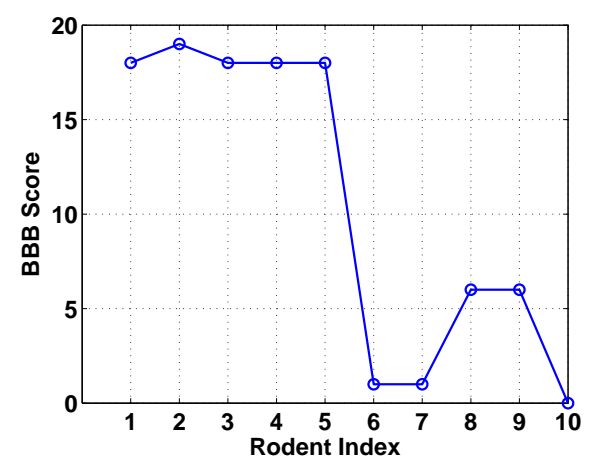

(c)

Fig. 5: (a) Impulse distance values using the SEP signals in Figure 3. in Figure 3. (c) BBB Test Values for rodent cohort.
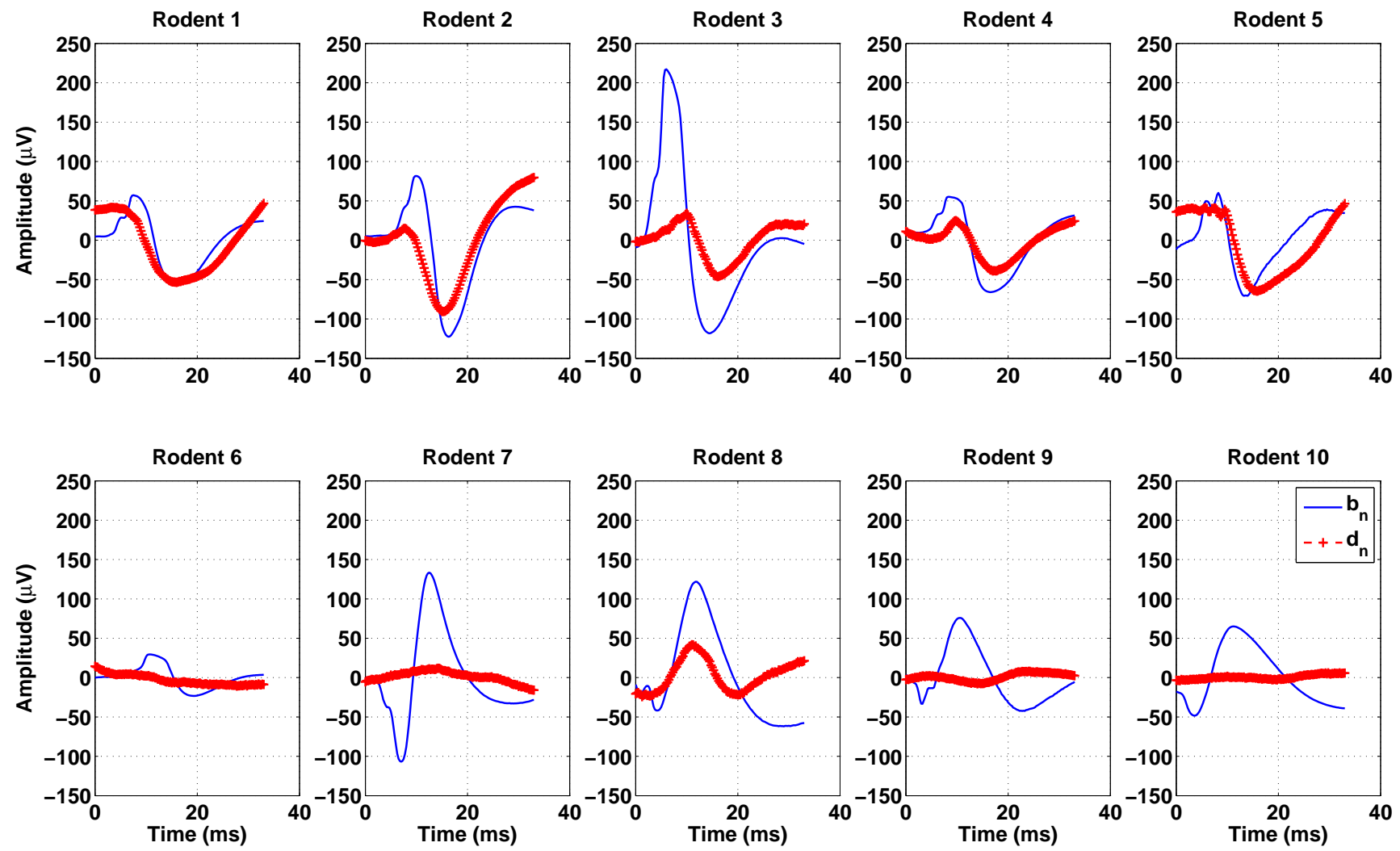

Fig. 6: Baseline forelimb SEP and SCI affected hindlimb SEP signal for rodents with spinal hemitransection (top row) and full spinal transection (bottom row). 

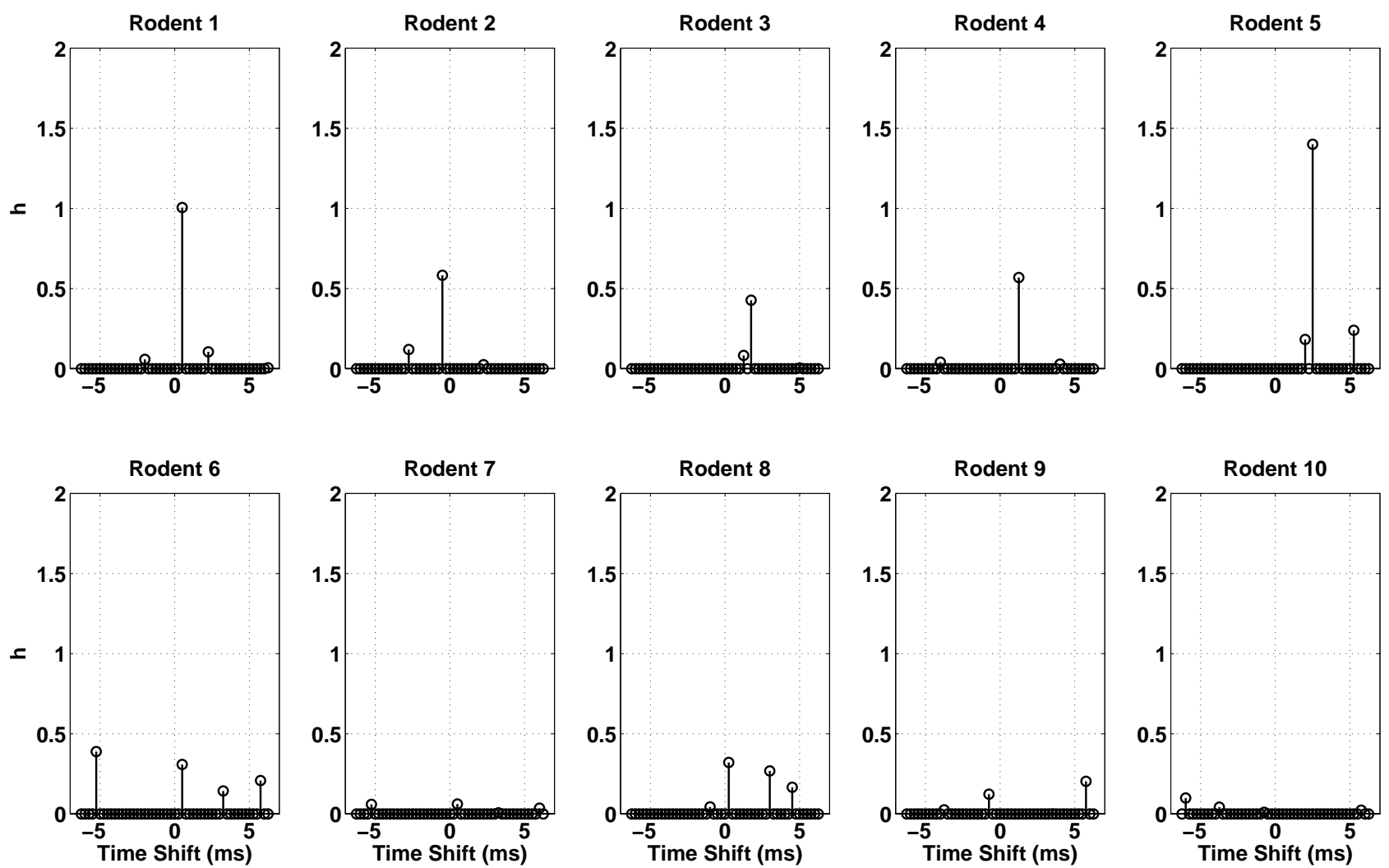

Fig. 7: Elements of $\mathbf{h}$ from solving (5) for rodents with spinal hemitransection (top row) and full spinal transection (bottom row) using the SEP signals in Figure 6.

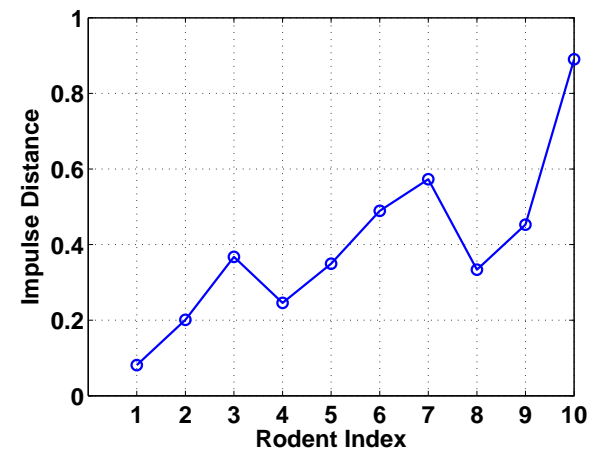

(a)

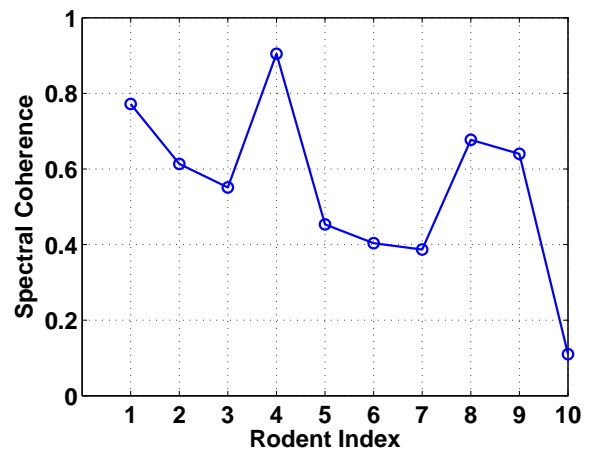

(b)

Fig. 8: (a) Impulse distance values using the SEP signals in Figure 6. in Figure 6.

(b) Spectral coherence values using the SEP signals

TABLE II: Pearson Correlation Coefficient magnitude for SCI assessment methods using the SEP signals in Figure 6

\begin{tabular}{|c|c|c|}
\hline & Spectral Coherence & BBB Score \\
\hline \hline Impulse Distance & 0.82 & 0.79 \\
\hline Spectral Coherence & 1 & 0.65 \\
\hline
\end{tabular}

gation velocity of an action potential. This results in a delayed SEP which also often has reduced amplitude. The proposed method accounts for both the amplitude and temporal aspects of the signal morphology, whereas spectral coherence accounts for only the amplitude characteristics. Indeed, the nature of the solution vector $\mathbf{h}$ provides a simplified representation of the SCI affected SEP signal vis-à-vis the baseline SEP signal, while the impulse distance measure further consolidates this representation as a single number. To illustrate the difference in the behavior of the spectral coherence and the proposed impulse distance measure, Figure 9 shows the resulting values of both measures for a temporal shift of the SCI affected SEP signal with respect the baseline SEP signal ranging from $0 \mathrm{~ms}$ to $3 \mathrm{~ms}$. It can be seen that the spectral coherence value is relatively insensitive across time shifts, whereas the impulse distance exhibits a more representative change in value. 
Finally, it may be noted that the impulse distance and spectral coherence are both quantitative assessment measures based on SEP signals that reflect the health of the sensory function, while the BBB reflects the status of the motor recovery. Moreover, various stages of sensory function may manifest as a similar level of motor skill. Hence, a quantitative measure of SCI such as the impulse distance coupled with a qualitative assessment such as the BBB may be viewed as complementary information that provides a more comprehensive assessment of SCI.
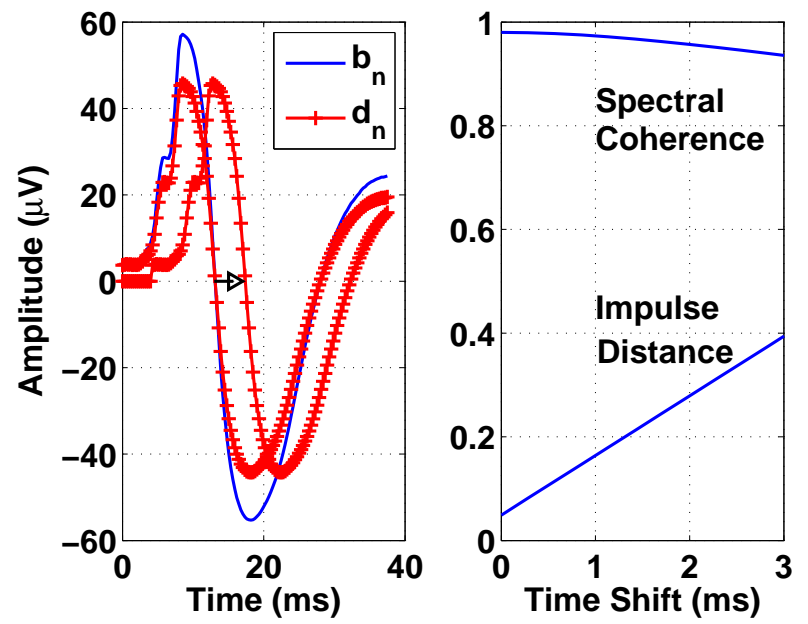

Fig. 9: Dependence of spectral coherence and impulse distance values on time shift between baseline and SCI affected SEP signals.

\section{CONCLUSION}

The similarity between the characteristics of SEP signals from a healthy spinal pathway and injured spinal cord pathway provides an indicator of the level of SCI. A method to assess the level of SCI was proposed that models the SEP signal from an injured pathway as a superposition of time-shifted and amplitude scaled versions of the SEP signal from a healthy spinal pathway. The time-shift and amplitude scaling parameters were determined by solving a sparse reconstruction problem. Using actual experimental data collected from rodents subject to SCI, it was shown for mild SCI, only a few significant elements located at time-shifts close to zero are needed to model the injury, whereas for severe SCI, many non-zero elements located at various time-shifts are required. The nature of the elements thus encapsulates information about the level of SCI. A measure was developed to express the elements as a single number, and it was shown that the values yielded by this measure are consistent with existing quantitative and qualitative SCI assessment methods, while simultaneously providing a closer inspection of morphological changes of the SEP signal induced by SCI.

\section{REFERENCES}

[1] F. Bazley. C. Hu, BS, A. Maybhate, A. Pourmorteza, N. Pashai, N Thakor, C. Kerr, and A. All, "Electrophysiological Evaluation of Sensory and Motor Pathways After Incomplete Unilateral Spinal Cord Contusion," Journal of Neurosurgery: Spine, vol. 16, pp. 414-423, Apr. 2012
[2] Spinal cord injury, Fact sheet $\mathrm{N}^{\circ} 384$, November 2013, http://www.who.int/mediacentre/factsheets/fs384/en/

[3] F. Bazley, A. Maybhate, T. Seng, N. Thakor, C. Kerr, and A. All, "Enhancement of Bilateral Cortical Somatosensory Evoked Potentials to Intact Forelimb Stimulation Following Thoracic Contusion Spinal Cord Injury in Rats," IEEE Transactions on Neural Systems and Rehabilitation Engineering, vol. 22, pp. 1-12, Sep. 2014

[4] F. Bazley, A. All, N. Thakor, and A. Maybhate, "Plasticity Associated Changes in Cortical Somatosensory Evoked Potentials Following Spinal Cord Injury in Rats, Proceedings IEEE Eng Med Biol Soc., pp. 20052008, 2011

[5] W. Truccolo, "Trial-to-trial variability of cortical evoked responses: Implications for the analysis of funtional connectivity," Clinical Neurophysiology, vol. 113, pp. 206-226, 2002.

[6] M. Nuwer, "Spinal cord monitoring," Muscle Nerve, vol. 22, pp.16201630, 1999.

[7] M. Shepherd and M. Bracken, "Magnetic resonance imaging and neurological recovery in acute spinal cord injury: Observations from the National Acute Spinal Cord Injury Study 3," Spinal Cord, vol. 37, pp 833-837, 1999.

[8] O.A. Glenn and J. Barkovich, "Magnetic resonance imaging of the fetal brain and spine: An increasingly important tool in prenatal diagnosis: Part 2, American Journal of Neuroradiology, vol. 27, pp. 1807-1814, 2006.

[9] M. Nuwer, "Fundamentals of evoked potentials and common clinical applications today," Electroencephalography and Clinical Neurophysiology, vol. 106, pp.142-148, 1998.

[10] H. Al-Nashash et al, "Spinal Cord Injury Detection and Monitoring Using Spectral Coherence," IEEE Transactions on Biomedical Engineering, vol. 56, no. 8, pp. 1971-1979, Aug. 2009.

[11] D. Basso, M. Beattie, and J. Bresnahan, "A sensitive and reliable locomotor rating scale for open field testing in rats," Journal of Neurotrama, vol. 12, pp. 1-21, 1995.

[12] E. Candes and M. Wakin, "An introduction to compressive sampling, IEEE Signal Processing Magazine, vol. 25, pp. 2130, Mar. 2008.

[13] A. Gilbert, M. Strauss, J. Tropp, and R. Vershynin, "One sketch for all: fast algorithms for compressed sensing," Proceedings of the 39th Annual ACM symposium on Theory of Computing, pp. 237-246, 2007

[14] J. Tropp and A.Gilbert, "Signal recovery from random measurements via orthogonal matching pursuit" IEEE Transactions on Information Theory, vol. 53, pp. 4655-4666, Dec. 2007.

[15] D. Needell and J. Tropp, "CoSaMP: Iterative signal recovery from incomplete and inaccurate samples," Applied and Computational Harmonic Analysis, vol. 26, no. 3, pp. 301321, May 2009.

[16] M. Davenport, D. Needell, and M. Wakin, "Signal space CoSaMP for sparse recovery with redundant dictionaries, IEEE Transactions on Information Theory, vol. 59, no. 10, pp. 68206829, Oct. 2013.

[17] Online: http://www.hopkinsmedicine.org/healthlibrary/conditions/ physical 'medicine' and 'rehabilitation/spinal ' cord' injury ' $85, \mathrm{P} 01180$ / 